\title{
Erratum: Ultrafast optical manipulation of magnetic order [Rev. Mod. Phys. 82, 2731 (2010)]
}

\author{
Andrei Kirilyuk, Alexey V. Kimel, and Theo Rasing \\ (published 11 August 2016) \\ DOI: 10.1103/RevModPhys.88.039904
}

\begin{abstract}
Large parts of Sec. VI.A, "Inverse magneto-optical excitation of magnetization dynamics: Theory," consist of text and equations that are verbatim quotes, or very nearly so, of material in the paper by Gridnev (2008). Although this theory was developed in close collaboration with Dr. Gridnev and jointly published in Kalashnikova et al., Phys. Rev. Lett. 99, 167205 (2007) and Kalashnikova et al., Phys. Rev. B 78, 104301 (2008), we adopted the more compact and rigorous description given in Gridnev (2008) for this review. We regret that we did not identify these parts of our review as direct quotes, and we apologize to V. N. Gridnev and the readers of Reviews of Modern Physics for this oversight.
\end{abstract}

\section{REFERENCES}

Gridnev, V. N., 2008, Phys. Rev. B 77, 094426. 